\title{
Edge Elements, their Advantages and their Disadvantages
}

\author{
Gerrit Mur \\ Faculty of Electrical Engineering \\ Delft University of Technology \\ P.O. Box 5031, 2600GA Delft, The Netherlands
}

\begin{abstract}
In finite-element methods for solving electromagnetic field problems, the use of edge elements has become very popular. In fact, edge elements are often said to be a cure for many difficulties that are encountered and they are claimed to yield accurate results. In the present paper we analyse these claims for tetrahedral elements. In particular we compare two different types of "linear" edge elements and the classical linear nodal element. Comparisons for higher-order elements and elements defined on other elementary subdomains, for instance on hexahedra, run along the same lines and yield similar conclusions.
\end{abstract}

\section{INTRODUCTION}

In the finite-element modeling of electromagnetic fields both edge and nodal elements play an important role. Recently edge elements have gained an increase in popularity because they are believed to be a cure for many difficulties that are encountered when attempting to solve electromagnetic field problems using finite elements. In this paper we analyse and compare both edge and nodal elements. For topological reasons [1] we prefer to carry out this analysis for elements defined on tetrahedra (simplices in 3D). Comparisons for elements defined on other elementary subdomains, for instance on hexahedra, run along the same lines and yield similar conclusions. Another assumption we make in the main text of this paper is that the fields to be modeled are free of divergence. The more general case where divergent fields are included is discussed in a separate section.

In our analysis we ignore a third type of finite element that is encountered in electromagnetic field computations, the face (or facet) element. Face elements are especially suited to modeling electric or magnetic fluxes. Their relative unpopularity may be due to their inefficiency, each expansion function having a support of only two elementary subdomains.

\section{NodAl Elements AND Edge ELEMENTS}

In this section we discuss some accuracy-related properties of nodal and edge elements. As regards the degree

Manuscript received Novernber 1,1993. of approximation, we confine ourselves to elements that yield "linear" polynomial expansions. The meaning of the double quote symbols will become clear in the course of our discussion.

\section{A. Nodal elements}

Early finite-element methods for solving electromagnetic field problems $[2,3,4]$ used linear nodal elements with three unknowns in each node (Fig. 1), either for approximating a vector potential or for approximating the electric and/or the magnetic field strength directly. For approximating electric and/or the magnetic field strengths, nodal elements have the disadvantage that, when using them, all components are automatically continuous functions of the spatial variables, thus preventing the modeling of the field

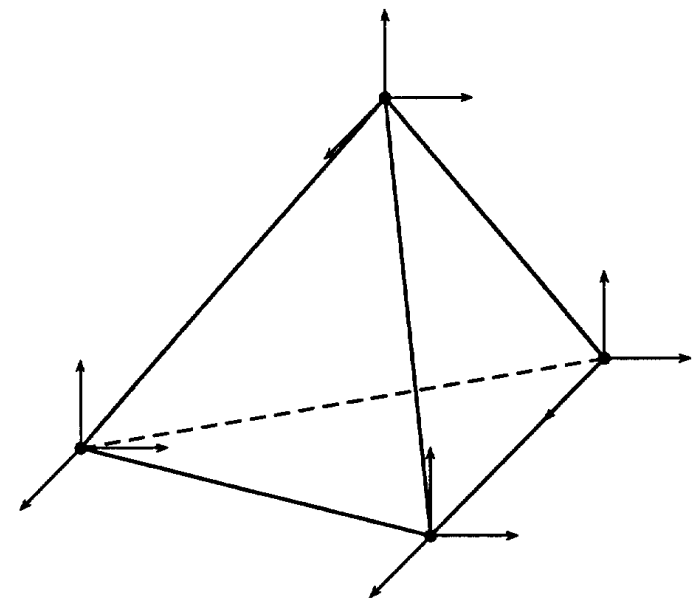

Fig. 1. Linear nodal element

when the properties of the media, and consequently (some components of) the electric and/or magnetic field strengths are discontinuous functions of the spatial coordinates. Trying to overcome this difficulty by introducing multiple nodes at the interfaces $[5,6]$ between different media causes difficulties at points where those interfaces are not locally flat. Nodal elements also cause difficulties near reentrant corners in the outer boundary of the domain of computation.

As regards their accuracy, nodal elements are consistently linear, i.e. each component of the vector field is rep- 
resented as a linear function of each coordinate direction. They yield local approximation errors of the order $O\left(h^{2}\right)$ [7], where $h$ denotes the largest dimension of the tetrahedron. Nodal elements have twelve unknowns in each tetrahedron.

\section{B. Edge elements}

The difficulty in the modeling of field strengths that is caused by discontinuities in the properties of the media can be overcome by using edge elements. Edge elements have the property that they ensure the continuity of tangential field components across an interface between different media, while leaving the normal field components free to jump across such interfaces. Because of this, edge elements can be used for modeling an electromagnetic field along interfaces between two different media. In our discussion of "linear" edge elements we investigate both Whitney 1 elements, which are of the mixed type, and consistently linear edge elements.

1) Edge elements (mixed type): The simplest elements of this type, and still the most popular ones, were proposed by Nédélec [8] (Fig.2). They have six unknowns in each tetrahedron, one attached to each edge. Nédélec's 'firstorder' edge elements, also called Whitney 1 elements, are mixed finite elements that have the additional property of being free of divergence.

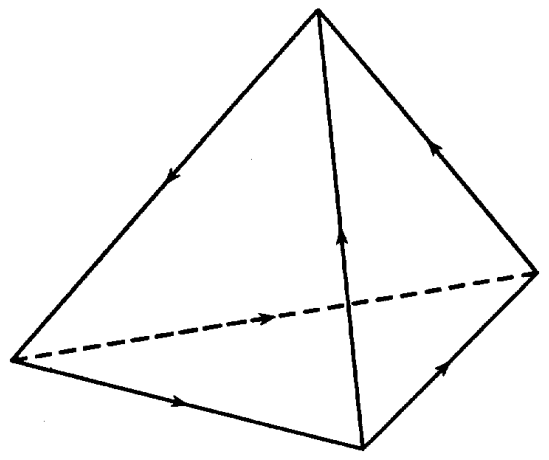

Fig. 2. Mixed "linear" edge element (Whitney 1)

Because of the fact that (surface) divergences may occur at the interfaces between adjoining elements (the normal component of the field is free to jump at each of the faces of the element), this freedom of divergence of the elements does not imply that fields computed using them are free of divergence. Therefore the continuity condition to be satisfied by the normal component of the field at the faces of edge elements should be made a part of the formulation of the problem. An additional disadvantage of these elements is that they are not consistently linear, they are constant in a certain spatial direction that follows from the generating edge and the geometry of the relevant tetrahedron while in other spatial directions they are linear functions. Because of being constant in a certain spatial direction, they yield local approximation errors of the order $O(h)$. For an experimental numerical verification of the fact that correspondingly large errors are found in global solutions $[9,10,11]$.

As regards the economy (numerical efficiency), the large local errors are not counterbalanced by the fact that only six unknowns are required for each tetrahedron. Note that the low degree of approximation is related to the fact that the element carries only six unknowns, one on each edge. The linear vectorial nodal element, or any other consistently linear 3D-vector function over a tetrahedron, carries twelve unknowns, three at each of its four vertices.

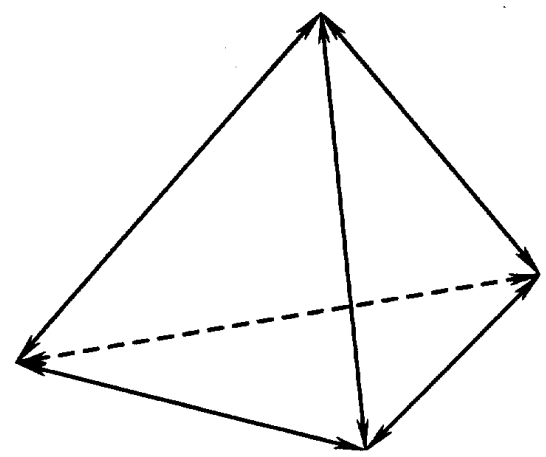

Fig. 3. Consistently linear edge element

2) Consistently linear edge elements: Edge elements that do not suffer from large local approximation errors are consistently linear edge elements (Fig. 3). These edge elements are linear vector functions of the position in all spatial directions and were first introduced in [9]. Consistently linear edge elements have twelve unknowns, two on each of their six edges. Like linear nodal elements they yield local approximation errors of the order $\mathrm{O}\left(h^{2}\right)$. Computationally they are, however, less efficient than linear nodal elements because of the fact that the span of global edge expansion functions is smaller than the span of global nodal expansion functions, which causes the edge elements to generate more unknowns for the same mesh.

3) Fields near objects with edges: Finally we mention that an additional advantage of edge elements, as compared with nodal elements, is provided by the fact that, when modeling the electric and/or magnetic field strength, they do not yield conflicting conditions at points where the interface between two different media is not locally flat. The use of edge elements near sharp edges of subdomains in the configuration, or near re-entrant corners in the outer boundary of the domain of computation, "relaxes" the continuity requirements of the fields near those edges. In fact, the difficulty caused by the attempt to model the field near a singularity by using linear elements 
is softened by the absence of strict normal continuity requirements at the interfaces between edge elements. Obviously this does not automatically imply that edge elements generate accurate results near singularities like edges in interfaces or re-entrant corners.

4) In summary: Consistently linear edge and nodal elements are more accurate $\left(O\left(h^{2}\right)\right)$ than (mixed) Whitney 1 elements $(O(h))$. As regards to applicability, we conclude that when using linear elements on tetrahedra, linear nodal elements can be used in homogeneous domains, edge elements, preferably consistently linear ones, should be used when discontinuities are encountered. Spurious solutions cannot, generally, be avoided by choosing (mixed) edge elements, they can only be precluded by choosing a proper formulation of the problem to be solved.

A detailed description the electromagnetic compatibility relations, which are the necessary conditions to be satisfied in the formulation, is given in [12].

\section{The Computational Costs}

When judging the relative numerical efficiency of edge elements versus nodal elements, it is not sufficient to compare their asymptotic rate of convergence. We also need to compare the number of free parameters and the sparsity of the system matrices. Kotiuga [13] presented an analysis of the the number of degrees of freedom and the number of nonzero entries in the matrices as a function of the number of nodes in the mesh. Using these results, explicit formulae for the number of FLOPS (floating point operations) per CG (conjugent gradient) iteration can be given. Kotiuga's results seem to indicate that, as regards computational efficiency, an edge based interpolation is to be preferred to a nodal interpolation.

Unfortunately, Kotiuga compares nodal elements, having a local approximation error of the order $\mathrm{O}\left(h^{2}\right)$, with mixed edge elements (Whitney 1 ) having a local approximation error of the order $O(h)$. Carrying out the same analysis for edge elements having a local approximation errors of the order $\mathrm{O}\left(h^{2}\right)$, which seems to be the proper thing to do, we find that the number of degrees of freedom doubles in comparison with Whitney 1 edge elements (compare Figs. 2 and 3 ). As regards the number of nonzero entries on a row of the matrices, we also find a doubling in comparison with Whitney 1 elements. Taking these two factors together, we conclude that matrices based on consistently linear edge elements contain four times as many nonzero entries as matrices based on Whitney 1 elements. Since the same factor four applies to the number of FLOPS per CG iteration, we find, using Kotiuga's notation as well as his analysis as regards the topology, the following numbers for a large mesh (i.e. a mesh large enough to neglect the effect of the boundaries in our analysis) with simple boundaries and having $M_{0}$ nodes.
1. $F_{0}^{H}=256 M_{0}$ for linear nodal interpolation on tetrahedra or hexahedra (local error $O\left(h^{2}\right)$ ),

2. $F_{1}^{T, 6}=150 M_{0}$ for edge interpolation using Whitney 1 elements with each hexahedron divided into 6 tetrahedra (local error $O(h)$ ),

3. $F_{1}^{T, 5}=126 M_{0}$ for edge interpolation using Whitney 1 elements with each hexahedron divided into 5 tetrahedra (local error $O(h)$ ),

4. $F_{\text {lin }}^{T, 6}=600 M_{0}$ for edge interpolation using consistently linear edge elements with each hexahedron divided into 6 tetrahedra (local error $O\left(h^{2}\right)$ ),

5. $F_{\text {lin }}^{T, 5}=504 M_{0}$ for edge interpolation using consistently linear edge elements with each hexahedron divided into 5 tetrahedra (local error $O\left(h^{2}\right)$ ).

Now, comparing the options that yield a local error $O\left(h^{2}\right)$, nodal elements are more efficient than edge elements by a factor of 2 or more. In many practical cases, the advantage of nodal elements over edge elements is even larger than the one following from the above analysis. This is for two reasons. The first is that because of the orthogonality of the Cartesian directions used in nodal elements, many nonzero matrix entries turn out to be zero, which can be used to optimize a node based code. The second is that imposing the continuity conditions at the interfaces between edge elements (the importance of which will become evident in the next section) increases the connectivity of the matrices significantly.

One of the reviewers suggested that, for obtaining a complete picture of the computational costs, the convergence characteristics of the CG iterations for nodal and edge elements should be included in the present analysis. Convergence of a CG method, however, strongly depends on the formulation of the problem and on the resulting condition of the system of equations to be solved. Provided that no extremely flat or elongated tetrahedra are used (see the next section) it does not, or much less, depend on the type of element used. Because of this, convergence characteristics of the CG iterations were not taken into account in our analysis.

As regards the storage requirements, we observe that they are, for all types of element included in the present analysis, proportional to the number of FLOPS per CG operation. Consequently, the conclusions based on storage requirements are identical to, and strengthen, those based on FLOPS per CG. Finally we note that the lower number of FLOPS per CG iteration step and the lower storage requirements for Whitney 1 elements do not counterbalance their poor asymptotic rate of convergence.

In summary: Linear edge elements are, both as regards the required number of FLOPS per CG operation and as regards the storage requirements, more than twice as expensive as linear nodal elements. Mixed edge elements are cheaper but cannot be compared because of their poor asymptotic rate of convergence. 


\section{THE CONDITION OF THE FIELD REPRESENTATION}

In a further comparison of nodal and edge elements we can observe the following. When representing a vector field with respect to a certain coordinate system it is advantageous, as regards the condition of the representation, to use a coordinate system with mutually perpendicular coordinate directions. Nodal elements use a Cartesian frame of reference and are therefore optimum in this respect. Edge elements use base vectors that follow from the orientation of the faces of the relevant tetrahedron. When the tetrahedron deviates strongly from the optimum one with equal edge lengths, these orientations are not mutually perpendicular. In fact, the base can be almost degenerate either because the base vectors are almost in one plane, or because (some of) the base vectors are almost in the same direction. In those cases large errors should be expected due to the ill condition of the representation of the vector field inside the tetrahedron and the resulting ill condition of the system matrices. These errors add to ill conditioning due to the use of extremely fiat or elongated (slender) elements [14].

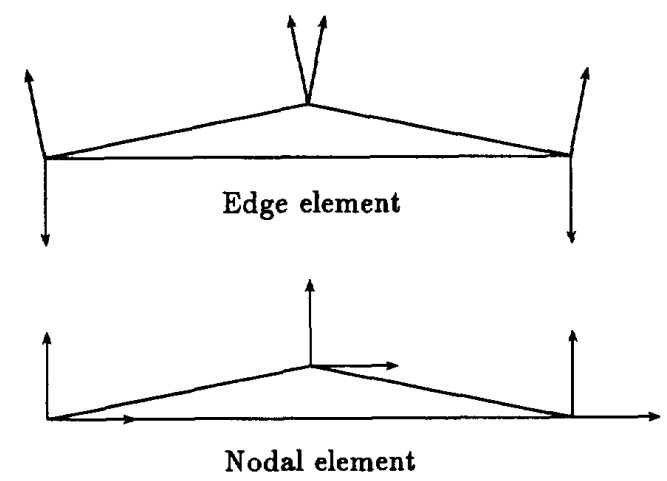

Fig. 4. Extremely flat triangular elements (2D) with bases of local coordinate systems

In Fig. 4 we have depicted two-dimensional examples of extremely flat triangular edge and nodal elements together with the local bases they employ. For the edge element, the consistently linear element is shown. Note the neardegenerate bases in each of its vertices and the orthogonal bases in the nodal element of the same shape. In linear edge elements the base vectors for the local coordinate systems in a given vertex of the tetrahedron are the outward normals to the faces that have that vertex in common. These base vectors constitute the base reciprocal to the base consisting of the directions of the edges having this vertex in common $[15,16]$, the latter base is used in face elements.

In summary: The condition of the field representa- tion deteriorates strongly when edge elements are used on tetrahedra that do not have approximately equal sidelengths. Nodal elements suffer less from these difficulties.

\section{EDgE ELEMENTS AND SPURIOUS SOLUTIONS}

A number of authors claim that edge elements have the advantage of not generating "spurious solutions" or "vector parasites" (in their type of problem) $[17,18,19,20,21$, $22,23]$. There is no doubt that these authors did not find any spurious solutions. The reason for that may be the formulation they used, the problem(s) they applied their elements to, or a combination of both. In our opinion, edge elements cannot be used to ensure the elimination of unwanted "solutions", they can only be used to eliminate one of their causes.

One reason why edge elements cannot be used for this purpose is related to one of their most important properties, the fact that they allow the normal component of the field that is represented to jump across the interface between adjacent elements. Edge elements do not in any way restrict the magnitude of the jump in the normal component and therefore this jump does not have to be in accordance with the local physical conditions. For instance, edge elements do not impose flux continuity if no surface charge is present at the inter element boundary. Consequently, when using them, the proper jump can only be obtained by making the relevant condition a part of the formulation of the problem.

An example: In this section we give an example demonstrating the fact that edge elements cannot be used for the elimination of unwanted "solutions".

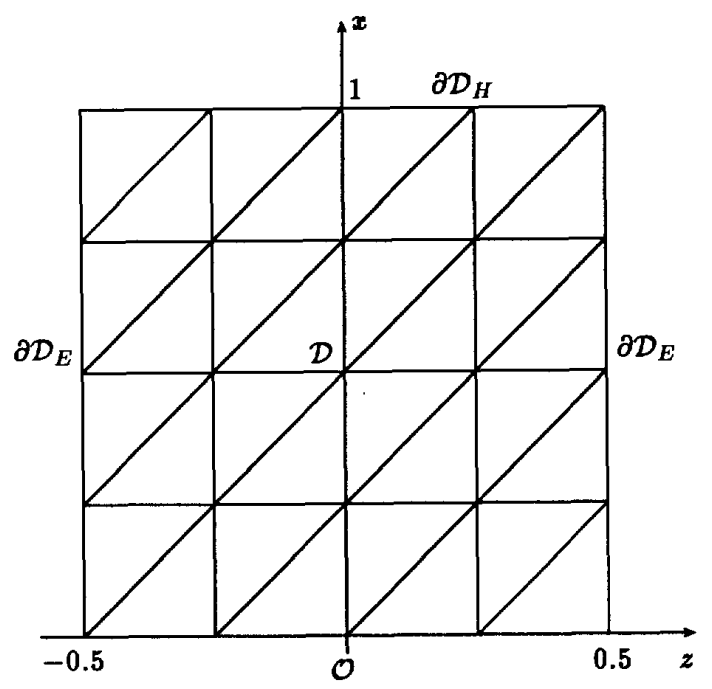

Fig. 5. Cube subdivided in tetrahedra, side view

In this example we assume a computational domain $\mathcal{D}$ with outer boundary $\partial \mathcal{D}$ in which we have a transient 
electromagnetic field $\{\boldsymbol{E}(\boldsymbol{r}, t), \boldsymbol{H}(\boldsymbol{r}, t)\}$. For the threedimensional domain $\mathcal{D}$, with a two-dimensional illustration as in Fig. 5 , we choose the cube $0 \leq x \leq 1,0 \leq y \leq 1$, $-0.5 \leq z \leq 0.5$. The lossy medium in this cube is assumed to be homogeneous with permittivity $\varepsilon=\varepsilon_{\mathrm{r}} \varepsilon_{0}$, permeability $\mu=\mu_{\mathrm{r}} \mu_{0}$ and conductivity $\sigma$, with $\varepsilon_{\mathrm{r}} \geq 1, \mu_{\mathrm{r}} \geq 1$ and $\sigma>0$. We assume the outer boundary $\partial \mathcal{D}$ to be divided into two parts (that may themselves be subdivided in a number of subdomains) viz. $\partial \mathcal{D}_{E}$ and $\partial \mathcal{D}_{H}$, where $\partial \mathcal{D}=\partial \mathcal{D}_{E} \cup \partial \mathcal{D}_{H} . \partial \mathcal{D}_{E}$ consists of those parts of $\partial \mathcal{D}$ that are located in one of the planes $z=-0.5$ or $z=0.5, \partial D_{H}$ is defined as the remaining part of $\partial \mathcal{D}$ i.e. $\partial \mathcal{D}_{H}=\partial \mathcal{D} \backslash \partial \mathcal{D}_{E}$.

We now assume that the tangential components of the electric field strength $\boldsymbol{E}(\boldsymbol{r}, t)$ are are known functions of space and time on $\partial D_{E}$, and that the tangential components of the magnetic field strength $H(r, t)$ are are known functions of space and time on $\partial D_{H}$. In addition to this we assume that the source distributions and the initial conditions $\boldsymbol{E}\left(\boldsymbol{r}, t_{0}\right)$ and $\boldsymbol{H}\left(\boldsymbol{r}, t_{0}\right)$ are known functions of space (and time) in $\mathcal{D}$. With these data we have defined an electromagnetic field problem with a unique solution [16]. For generating a finite-element solution to this problem we assume the domain of computation to be covered by a uniform mesh consisting of identical cubes of sidelength $0.25 \mathrm{~m}$ (Fig. 5), each being subdivided into tetrahedra.

As regards the problem to be solved, we assume that, after a transient in time ending at $t=t_{\text {end }}>t_{0}$, the exact solution for the electric field strength has converged to

$$
\boldsymbol{E}(\boldsymbol{r}, t)=E_{0} i_{z}
$$

within an acceptable degree of accuracy. Since this solution is constant in both the spatial and the time coordinates, it can be represented exactly using edge elements of any degree, and there is no doubt that many methods using edge expansions will find this solution. The point we want to stress here is that when a correct solution is found its correctness can only be attributable to the finiteelement formulation used and not to the use of edge elements. This is most easily understood by verifying that the "solution"

$$
\begin{array}{rll}
=E_{0} i_{z}, & \text { for }-0.50<z<-0.25 \\
E(r, t) & =0 \dot{i}_{z}, & \text { for }-0.25<z<0.25 \\
=E_{0} i_{z}, & \text { for } 0.25<z<0.50
\end{array}
$$

for $t \geq t_{\text {end }}$, is wrong or "spurious". However, since the errors in the above wrong solution consist of jumps in its normal component across the inter element boundaries at the planes $|z|=0.25$ it is an admissable solution when judging this from the properties of the edge elements. At the inter-element planes the normal component of the exact solution is continuous. Only a correct formulation of the problem could have prevented the unwanted discontinuity in the normal components from entering into the "solution".

Finally note that the fact that our example discusses a transient to a static solution is immaterial. The example was chosen for the sake of clarity and simplicity. Any other behavior in time could have been chosen instead in (1) and (2). Accurate, non-spurious, solutions can only be guaranteed by making the continuity of the normal component of the flux between edge elements a part of the formulation of the finite-element method.

In summary: We have presented an example illustrating the fact that spurious solutions cannot be made impossible by using edge elements.

\section{NONZERO DIVERGENCES}

In some cases, for instance when the sources of the electromagnetic field or the initial conditions are not free of divergence, the exact solution of an electromagnetic field problem is not free of divergence. Obviously, Whitney 1 elements cannot be used in those cases. They would force the divergence of solution to be zero inside each tetrahedron, and a choice has to be made between nodal elements and consistently linear edge elements. When making a choice between them, the conclusions of the earlier sections of this paper for solutions having zero divergence remain valid. Of course the finite-element formulation of the problem has to be such that the proper divergence conditions are included in it [12].

\section{Edge Elements And Vector Potentials}

Edge and nodal elements are frequently used in finite element methods for solving (electro)magnetic field problems using vector potentials [24]. The results of our analysis regarding the comparison of these types of element can be applied directly to vector potential methods for solving field problems. Note that, when doing so, the asymptotic estimates of the local error apply to the vector potential only and not to the electric and/or magnetic fields derived from those potentials because the evaluation of the latter quantities usually requires a numerical differentiation with an accompanying loss in the asymptotic order of accuracy. No convergence studies for vector potential methods involving problems with analytic solutions and comparing edge and nodal elements are known to the author. There is no reason to assume that the rates of convergence in terms of vector potentials would differ significantly from those obtained earlier for field strengths $[9,10]$.

\section{CONCLUSIONS}

Edge elements can be used for computing electromagnetic fields in both homogeneous and inhomogeneous domains. They are an indispensable tool for the modeling 
of electromagnetic fields along interfaces between regions with different medium properties. Nodal elements can be used in homogeneous (sub)domains only. Neither nodal elements nor edge elements cause the solution to satisfy the correct divergence condition, or putting this in more general terms, to satisfy the electromagnetic compatibility relations [12]. In other words, none of these elements guarantees that the solution obtained will be free of "spurious solutions" and "vector parasites". Consequently, numerical errors of this type can only be eliminated by choosing a problem formulation that includes Maxwell's equations together with its compatibility relations. As regards the accuracy, consistently linear (edge or nodal) elements are preferable because of the fact that they have a higher asymptotic rate of convergence than (mixed) Whitney 1 elements.

The main advantages of edge elements are that they facilitate the modeling of the field near a "singularity", i.e. they allow normal field components to jump across interfaces and that they do not yield conflicting conditions near edges of subdomains with contrasting medium properties and near re-entrant corners. Away from those "singularities" nodal elements are always preferable because of their higher efficiency.

\section{ACKNOWLEDGMENT}

The research reported in this paper has been financially supported through Research Grants from the Stichting Fund for Science, Technology and Research (a companion organization to the Schlumberger Foundation in the U.S.A.), from Schlumberger-Doll Research, Ridgefield, CT, U.S.A., from Etudes et Productions Schlumberger, Clamart, France, and from Schlumberger Cambridge Research Limited, Cambridge, England. This support is gratefully acknowledged.

\section{REFERENCES}

[1] G. L. Naber, Topological Methods in Euclidean Spaces, Cambridge, Cambridge University Press, 1980.

[2] M. V. K. Chari, A. Konrad, M. A. Palmo and J. D'Angelo, "Three-dimensional vector potential analysis for machine field problems," IEEE Trans, on Magnetics, vol. MAG-18, no. 2, pp. 436-446, March 1982.

[3] M. E. Best, P. Duncan, F. J. Jacobs and W. L. Scheen, "Numerical modeling of the electromagnetic response of threedimensional conductors in a layered earth," Geophysics, vol. 50, pp. 665-676, April 1985.

[4] G. W. Hohmann, "Three-dimensional EM modeling," Geophys ical Surveys, vol. 6, pp. 27-53, 1983.

[5] M. Koshiba, K. Hayata and M. Suzuki, "Finite-element formulation of the electric-field vector for electromagnetic waveguide problems," IEEE Trans. on Microwave Theory and Techniques, vol. MTT-33, 1985, pp. 900-905.

[6] D. R. Lynch and K. D. Paulsen, "Time-domain integration of the Maxwell equations on finite elements," IEEE Trans. on Antennas and Propagation, vol. AP-38, pp. 1933-1942, Decemder 1990.

[7] G. Strang and G. J. Fix, An Analysis of the Finile Element Method, Englewood Cliffs, Prentice-Hall, 1973.
[8] J.C. Nédélec, "Mixed finite elements in $\mathbf{R}^{3}$," Numer. Math., vol. 35, pp. 31 5-341, 1980.

[9] G. Mur and A. T. de Hoop, "A finite-element method for computing three-dimensional electromagnetic fields in inhomogeneous media," IEEE Trans. on Magnetics, vol. MAG-21, no. 6, pp. 2188-2191, November 1985.

[10] B. Bandelier and F. Rioux-Damidau, "Modelling of magnetic fields using nodal or edge variables," IEEE Trans. on Magnetics, vol. MAG-26, no. 5, pp. 1644-1646, September 1990.

[11] H. Trabelsi, F. Rioux-Damidau and B. Bandelier , "Finite element 3D modelling of electromagnetic fields with tetrahedral and hexahedral elements," J. Phys. III, France, vol. 2, pp. 20692081, November, 1992.

[12] G. Mur, "Compatibility relations and the finite-element formulation of electromagnetic field problems," IEEE Trans. on Magnetics, this issue.

[13] P. R. Kotiuga, "Essential arithmetic for evaluating three dimensional vector finite element interpolation schemes," IEEE Trans. on Magnetics, vol. MAG-27, no. 6, pp. 5208-5210, November 1991.

[14] G. F. Carey and J. T. Oden, Finite Elements, Computational Aspects, vol. III, Englewood Cliffs, Prentice-Hall, 1984.

[15] L. Brillouin, Wave Propagation in Periodic Structures, Dover, 1953, p. 131.

[16] J. A. Stratton, Electromagnetic Theory, McGraw-Hill Book Company, New York, 1941, p. 486.

[17] J. P. Webb, "Edge elements and what they can do for you," IEEE Trans. on Magnetics, vol. MAG-29, no. 2, pp. 1460-1465, March 1993.

[18] A. Bossavit, "Solving Maxwell equations in a closed cavity, and the question of spurious modes," IEEE Trans. on Magnetics, vol. MAG-26, no. 2, pp. 702-705, March 1990.

[19] A. Bossavit and I. Mayergoyz, "Edge-elements for scattering problems," IEEE Trans. on Magnetics, vol. MAG-25, no. 4, pp. 2816-2821, July 1989.

[20] J. D'Angelo and I. D. Mayergoyz, "Three dimensional RF scattering by the finite element method," IEEE Trans. on Magnetics, vol. MAG-27, no. 5, pp. 3827-3832, September 1991.

[21] J.-S. Wang and N. Ida, "Eigenvalue analysis in electromagnetic cavities using divergence free finite elements," IEEE Trans. on Magnetics, vol. MAG-27, no. 5, pp. 3978-3981, September 1991.

[22] Z. J. Cendes, "Vector finite elements for electromagnetic field computation," IEEE Trans. on Magnetics, vol. MAG-27, no. 5, pp. 3958-3966, September 1991.

[23] M. Hano, "Vector finite-element solution of anisotropic waveguides using novel triangular elements," Electronics and Communications in Japan, pt. 2, vol. 71, no. 8, pp. 71-80, 1988

[24] K. Preis, I. Bardi, O. Biro, C. Mangele, G. Vrisk, and K. R. Richter, "Different finite element formulations of 3D magnetostatic fields," IEEE Trans. on Magnetics, vol. MAG-28, no. 2, pp. 1056-1059, March 1992. 